\title{
Publisher's Note: Optical Conductivity of Topological Surface States with Emergent Supersymmetry [Phys. Rev. Lett. 116, 100402 (2016)]
}

William Witczak-Krempa and Joseph Maciejko

(Received 16 September 2016; published 29 September 2016)

DOI: 10.1103/PhysRevLett.117.149903

This Letter was published online on 11 March 2016 with an omission in the Acknowledgments. The last sentence of the Acknowledgments should read as "This research was also supported in part by the National Science Foundation under Grant No. NSF PHY11-25915 (KITP) and by MURI Grant No. W911NF-14-1-0003 from ARO.” The Acknowledgments have been corrected as of 20 September 2016. The Acknowledgments are incorrect in the printed version of the journal. 